\title{
Hypermethylation of DAPK1 is an independent prognostic factor predicting survival in diffuse large B-cell lymphoma
}

\author{
Lasse Sommer Kristensen ${ }^{1}$, Fazila Asmar ${ }^{1}$, Konstantinos Dimopoulos ${ }^{1}$, Mette \\ Kathrine Nygaard², Derya Aslan'1, Jakob Werner Hansen ${ }^{1}$, Elisabeth Ralfkiaer ${ }^{3}$, \\ Kirsten Grønbæk ${ }^{1}$ \\ ${ }^{1}$ Department of Haematology, Rigshospitalet, Blegdamsvej 9, 2100-DK, Copenhagen, Denmark \\ ${ }^{2}$ Department of Haematology, Aalborg University Hospital, Mølleparkvej 4, 9000-DK, Aalborg, Denmark \\ ${ }^{3}$ Department of Pathology, Rigshospitalet, Copenhagen, Denmark \\ Correspondence to: \\ Lasse Sommer Kristensen, e-mail: lasse.sommer.kristensen@regionh.dk \\ Keywords: Diffuse Large B-Cell Lymphoma, Rituximab, prognostic markers, allele-specific DNA methylation, DAPK, DAPK1, \\ mutations, TP53 \\ Received: August 20, 2014 \\ Accepted: August 21, 2014 \\ Published: November 12, 2014
}

\section{ABSTRACT}

Diffuse large B-cell lymphoma (DLBCL) is the most common type of nonHodgkin's Iymphoma. Improvements in overall survival have been observed with the introduction of rituximab in combination with cyclophosphamide, doxorubicin, vincristine, and prednisone (R-CHOP), however, prognostic markers are still needed. Methylation of the death associated protein kinase (DAPK or DAPK1) gene and TP53 mutations are likely to have prognostic value in DLBCL. We have assessed TP53 mutations and allelic DAPK1 methylation patterns in a cohort of 119 DLBCL patients uniformly treated with R-CHOP-like regimens. We found that DAPK1 promoter methylation was associated with shorter overall survival $(p=0.017)$ and diseasespecific survival $(p=0.023)$. In multivariate analyses DAPK1 methylation remained as an independent prognostic factor predicting disease-specific survival $(p=0.038)$. When only considering individuals heterozygous for the rs13300553 SNP monoallelic methylation of the A-allele was associated with shorter overall- and disease-specific survival $(p<0.001)$. Patients carrying both DAPK1 methylation and a TP53 mutation had an inferior survival compared to patients carrying only one of these molecular alterations, however, this was borderline statistically significant. Allele-specific DAPK1 methylation patterns were also studied in a cohort of 67 multiple myeloma patients, and all of the methylated multiple myeloma samples heterozygous for the rs 13300553 SNP were methylated on both alleles.

\section{INTRODUCTION}

Improvements in overall survival of diffuse large B-cell lymphoma (DLBCL) patients have been observed with the introduction of rituximab in combination with cyclophosphamide, doxorubicin, vincristine, and prednisone (R-CHOP), however, prognostic markers are still needed [1]. Methylation of the death associated protein kinase (DAPK or DAPK1) gene and TP53 mutations are likely to have prognostic value in DLBCL, and a better understanding of the molecular pathways leading to DLBCL progression may be important for the development of novel therapies aiming at causing DLBCL cells to undergo apoptosis [2].

DAP-kinase (DAPK or DAPK1) is a serine/ threonine kinase that has a calcium/calmodulin activated autoregulatory domain in its N-terminus. In addition, DAPK1 has a number of extra-catalytic domains, including ankyrin repeats and a death domain, which facilitate interactions with numerous other proteins [3]. Many of these proteins have been implicated in cancer. Most prominent is $\mathrm{p} 53$, which is both an indirect and direct substrate of DAPK1. The indirect mechanism of DAPK1 dependent p53-activation is through activation of the ARF tumour 
suppressor, which inhibits MDM2, an inhibitor of p53. The direct mechanism is through DAPK1 phosphorylation of tetrameric p53 on Ser20, which is located within the transactivation domain that binds p300, leading to p53 activation and apoptosis $[4,5]$. In addition, the $D A P K 1$ gene is a transcriptional target of p53 and, therefore, may be part of a positive feedback loop controlling p53 activation and apoptosis [6]. However, DAPK1 may also facilitate apoptosis independent of $\mathrm{p} 53$, and is an essential component in several cell death signalling pathways (Figure 1). Because of its ability to sensitize cells to many of the apoptotic signals that are encountered during malignant transformation $D A P K 1$ is considered to be a tumour suppressor gene [7].

$D A P K 1$ has also been shown to be regulated at the transcriptional and translational levels by methylation of its promoter $\mathrm{CpG}$ island and by microRNAs, respectively [8]. In several haematological malignancies, including DLBCL, DAPK1 undergoes DNA methylation-mediated silencing during tumorigenesis. The frequency of $D A P K 1$ methylation in DLBCL patients is relatively high, but varies somewhat from study to study [9-12]. We have previously shown that almost $90 \%$ of DLBCL patients have detectable $D A P K 1$ methylation [13]. Some controversy exists in the literature whether or not $D A P K 1$ methylation is a prognostic factor in DLBCL [10-13]. This may be explained by the studied cohorts being small and/or not uniformly treated.

Mutations in the TP53 gene have been shown to confer a negative effect on survival in DLBCL [14]. Moreover, several studies have shown that TP53 disruption in combination with other molecular alterations such as deletion of the INK4a/ARF locus at chromosome 9p21 or $M I R 34 A$ promoter methylation, are associated with exceedingly poor prognosis [15-17].

A variety of different methods are available for DNA methylation studies, all having inherent strengths and weaknesses $[18,19]$. However, the vast majority does not evaluate allelic methylation patterns. Hence, only very few studies have investigated allelic methylation patterns of tumour suppressor genes in cancer. We, and others, have previously shown that validation of methylationspecific PCR (MSP) products by pyrosequencing provides a sensitive and specific method for the study of methylation $[13,20]$. In addition, we designed our $D A P K 1$ methylation assay to allow allele-specific methylation information to be obtained, as we hypothesized that biallelic methylation of $D A P K 1$ is a more severe event compared to monoallelic methylation.

In this contribution, we have increased a previously studied cohort [13] to 119 patients uniformly treated with R-CHOP-like regimens and increased the follow-up time. In addition to allelic $D A P K 1$ methylation patterns, mutation status of the TP53 gene was evaluated. Potential correlation between DAPK1 methylation and TP53 mutations was investigated. Effects on overall survival and disease-specific survival were investigated for DAPK1 methylation, $D A P K 1$ allelic methylation patterns, and TP53 mutations, alone or in combination. Allele-specific expression of DAPK1 mRNA was studied in a subset of the samples heterozygous for the rs3818584 SNP. In addition, allelic $D A P K 1$ methylation patterns were studied in a cohort of 67 multiple myeloma patients.

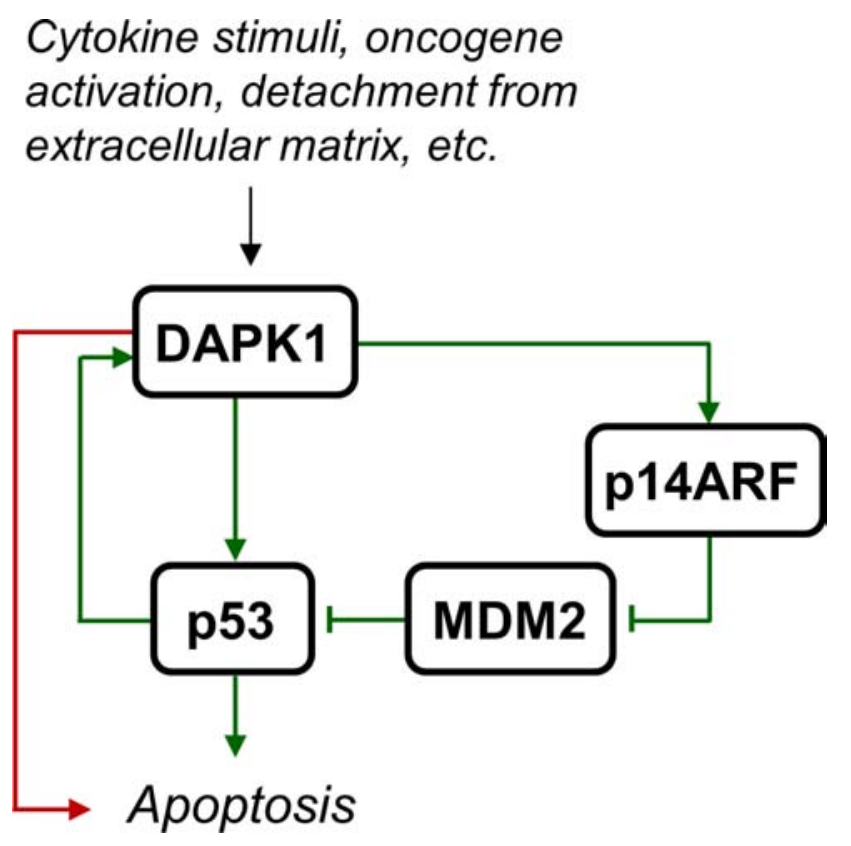

Figure 1: DAPK1 activation leads to apoptosis. Various stimuli such as cytokine signalling (e.g. Fas, IFN- $\gamma$, and TNF- $\alpha$ ), oncogene activation, and detachment from extracellular matrix, may lead to DAPK1 activation, which leads to apoptosis independent of p53 (red arrow) or through direct or indirect activation of p53 (green arrows). 


\section{RESULTS}

\section{$D A P K 1$ methylation status according to patient characteristics}

The clinical characteristics of the DLBCL patients as a function of $D A P K 1$ methylation status are shown in Table 1. No significant differences in patient or disease characteristics according to DAPK1 methylation status were observed, with the exception of age, which was borderline significant. This was also the case when analysing clinical characteristics of patients who were methylated and heterozygous for the rs $13300553 \mathrm{SNP}$ as a function of monoallelic DAPK1 methylation of the A-allele (data not shown), with three exceptions: elevated LDH (more patients with monoallelic DAPK1 methylation of the A-allele had elevated LDH, $p=0.007$ ), sex (more patients with monoallelic $D A P K 1$ methylation of the A-allele were male, $p=0.008$ ), and performance score (more patients with monoallelic $D A P K 1$ methylation of the A-allele had a poor performance, $p=0.047$ ). The mean age of the patients with monoallelic $D A P K 1$ methylation of the A-allele was 62.36 years and 60.42 years for the patients having the other possible allelic methylation patterns $(p=0.647)$.

Table 1: Clinical characteristics of the DLBCL patients as a function of DAPK1 methylation status.

\begin{tabular}{|c|c|c|c|c|}
\hline & Total $(n=119)$ & Unmethylated $(n=17)$ & Methylated (n=102) & $p$-value \\
\hline \multicolumn{5}{|l|}{ Sex } \\
\hline Men & 58 & 8 & 50 & \multirow{2}{*}{0.88} \\
\hline Women & 61 & 9 & 52 & \\
\hline \multicolumn{5}{|c|}{ Extranodal involvement } \\
\hline Yes & 57 & 8 & 49 & \multirow{2}{*}{0.94} \\
\hline No & 62 & 9 & 53 & \\
\hline \multicolumn{5}{|l|}{ Stage } \\
\hline I-II & 51 & 8 & 43 & \multirow{2}{*}{0.71} \\
\hline III-IV & 68 & 9 & 59 & \\
\hline \multicolumn{5}{|l|}{ Elevated LDH } \\
\hline Yes & 58 & 10 & 48 & \multirow{2}{*}{0.37} \\
\hline No & 61 & 7 & 54 & \\
\hline \multicolumn{5}{|l|}{ B symptoms } \\
\hline Yes & 48 & 5 & 43 & \multirow{2}{*}{0.32} \\
\hline No & 71 & 12 & 59 & \\
\hline \multicolumn{5}{|l|}{ IPI score } \\
\hline $0-2$ & 80 & 12 & 68 & \multirow{2}{*}{0.75} \\
\hline $3-5$ & 39 & 5 & 34 & \\
\hline \multicolumn{5}{|c|}{ Performance score* } \\
\hline $0-2$ & 113 & 17 & 96 & \multirow{2}{*}{0.59} \\
\hline $3-5$ & 6 & 0 & 6 & \\
\hline \multicolumn{5}{|l|}{ Response } \\
\hline $\mathrm{CR} / \mathrm{Cru}$ & 98 & 16 & 82 & \multirow{3}{*}{0.36} \\
\hline $\mathrm{PD} / \mathrm{PR}$ & 11 & 0 & 11 & \\
\hline Missing/Mors & 10 & 1 & 9 & \\
\hline
\end{tabular}

(Continued) 


\begin{tabular}{|l|c|c|c|c|}
\hline \multicolumn{2}{c}{ Total $(\mathbf{n}=\mathbf{1 1 9})$} & \multicolumn{1}{c}{ Unmethylated $(\mathbf{n}=\mathbf{1 7})$} & Methylated $(\mathbf{n}=102)$ & $\boldsymbol{p}$-value \\
\hline Age at diagnosis & & & & \\
\hline Below 65 years & 67 & 11 & 56 & \multirow{2}{*}{0.45} \\
\hline Above 65 years & 52 & 6 & 46 & 0.04 \\
\hline Age, mean (range) & $59.8(22-90)$ & 53.1 years $(28-77)$ & 60.7 years $(22-90)$ & 0.04 \\
\hline
\end{tabular}

*Eastern Cooperative Oncology Group

$\mathrm{LDH}$, lactate dehydrogenase; IPI, international prognostic index; CR, complete response, unconfirmed complete response; $\mathrm{PD}$, progressive disease; PR, partial response.

\section{Overall- and disease-specific survival analysis according to $D A P K 1$ methylation}

One-hundred and two of the 119 DLBCL patient samples analysed were methylated (85.7\%). Only one of the patients having an unmethylated tumour sample died during the follow-up period. Thus, DAPK1 methylation was associated with shorter overall survival (Figure 2A) and disease-specific survival (Figure 2B). Apparently, this association was not statistically significant in our previous study [13] due to a limited sample size and follow-up time.

\section{Overall- and disease-specific survival analysis according to allelic $D A P K 1$ methylation patterns}

Genotyping of the rs 13300553 SNP was successful for 109 of the samples. No association was observed between individual genotypes and overall- or disease specific survival in this study (Supplementary Figure 1A and 1B). Fifty-eight of the patients were heterozygous, and pyrosequencing was used to assess their allelic methylation patterns. This was successful for 57 of the samples. Twenty-five were methylated on both alleles, 13 were methylated only on the G-allele, 11 were methylated only on the A-allele, and eight were unmethylated (representative results are shown in Figure 3). It was observed that monoallelic methylation of the A-allele was associated with shorter overall survival (Figure 4C) and disease-specific survival (Figure 4D). There was no difference in survival between patients methylated on the G-allele and patients methylated on both alleles (Supplementary Figure 2A and 2B). In addition, there was no difference in survival between patients being methylated and homozygous AA and patients being methylated and homozygous GG (data not shown).

\section{Multivariate analysis of disease-specific survival according to $D A P K 1$ methylation}

Univariate analysis revealed that disease-specific survival was also significantly shortened according to the following parameters: stage, elevated LDH, B symptoms, high IPI score, performance score, and age at diagnosis (Table 2). Therefore, these baseline risk factors were included in the Cox proportional hazard model (Table 3 ). The model identified methylation of DAPK1 as an independent prognostic factor for predicting disease-specific survival, in addition to elevated $\mathrm{LDH}$. The hazard ratio was larger for $D A P K 1$ methylation $(8.4,95 \%$ CI was 1.13 to 62.88$)$ than for elevated $\mathrm{LDH}(4.58,95 \% \mathrm{CI}$ was 1.78 to 11.83$)$, but the confidence interval was also wider for DAPK1 methylation.

\section{Multivariate analysis of disease-specific survival according to monoallelic methylation of the A-allele}

This analysis was performed for the 49 DLBCL patients who were methylated and heterozygous for the rs13300553 SNP. In this patient group univariate analysis revealed that disease-specific survival was significantly shortened in patients with elevated LDH $(p<0.001)$ and high IPI score $(p=0.001)$. Therefore, these two baseline risk factors were included in the Cox proportional hazard model. In the multivariate analysis only elevated $\mathrm{LDH}$ remained as an independent factor predicting disease-specific survival. On the other hand IPI score and monoallelic methylation of the A-allele did not stand out as independent prognostic factors in this patient group (Table 3).

\section{Allele-specific- and quantitative expression analyses of $D A P K 1$}

DAPK1 expression levels and allele-specific expression were assessed for 24 DLBCL samples. Fourteen of these samples were heterozygous for the rs 3818584 SNP used to assess allele-specific expression. All fourteen of these samples clearly expressed both alleles as assessed by HRM (representative results are shown in Figure 5A). Eight random samples were selected for confirmation of the HRM results by pyrosequencing, and these analyses gave the same result for all samples (data not shown). Nine of the samples heterozygous for the rs3818584 SNP were also heterozygous for the rs 13300553 SNP used to assess allelic methylation patterns, and three of these were methylated only on one allele. Overall, sixteen of the 24 samples were 

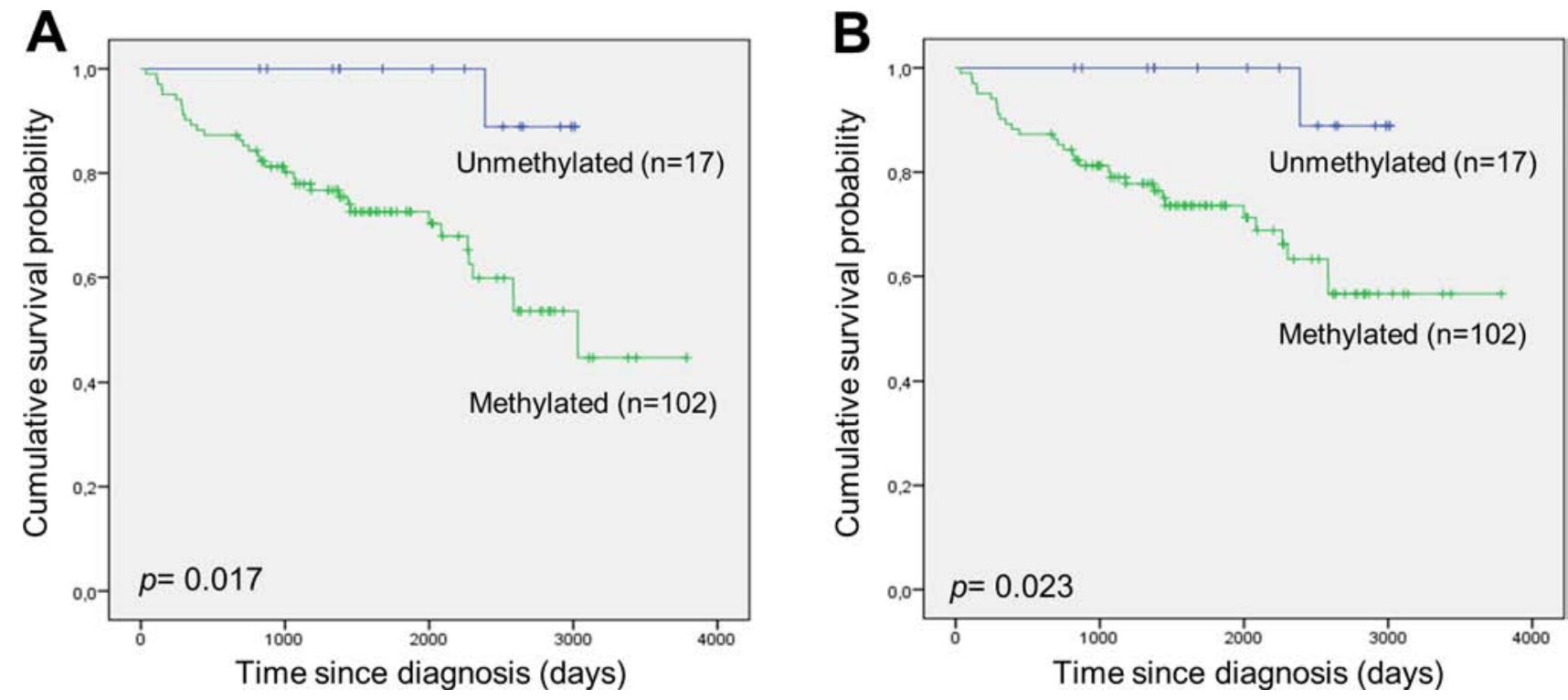

Figure 2: DAPK1 methylation and survival in DLBCL. (A) Overall survival according to DAPK1 methylation. (B) Diseasespecific survival according to DAPK1 methylation.

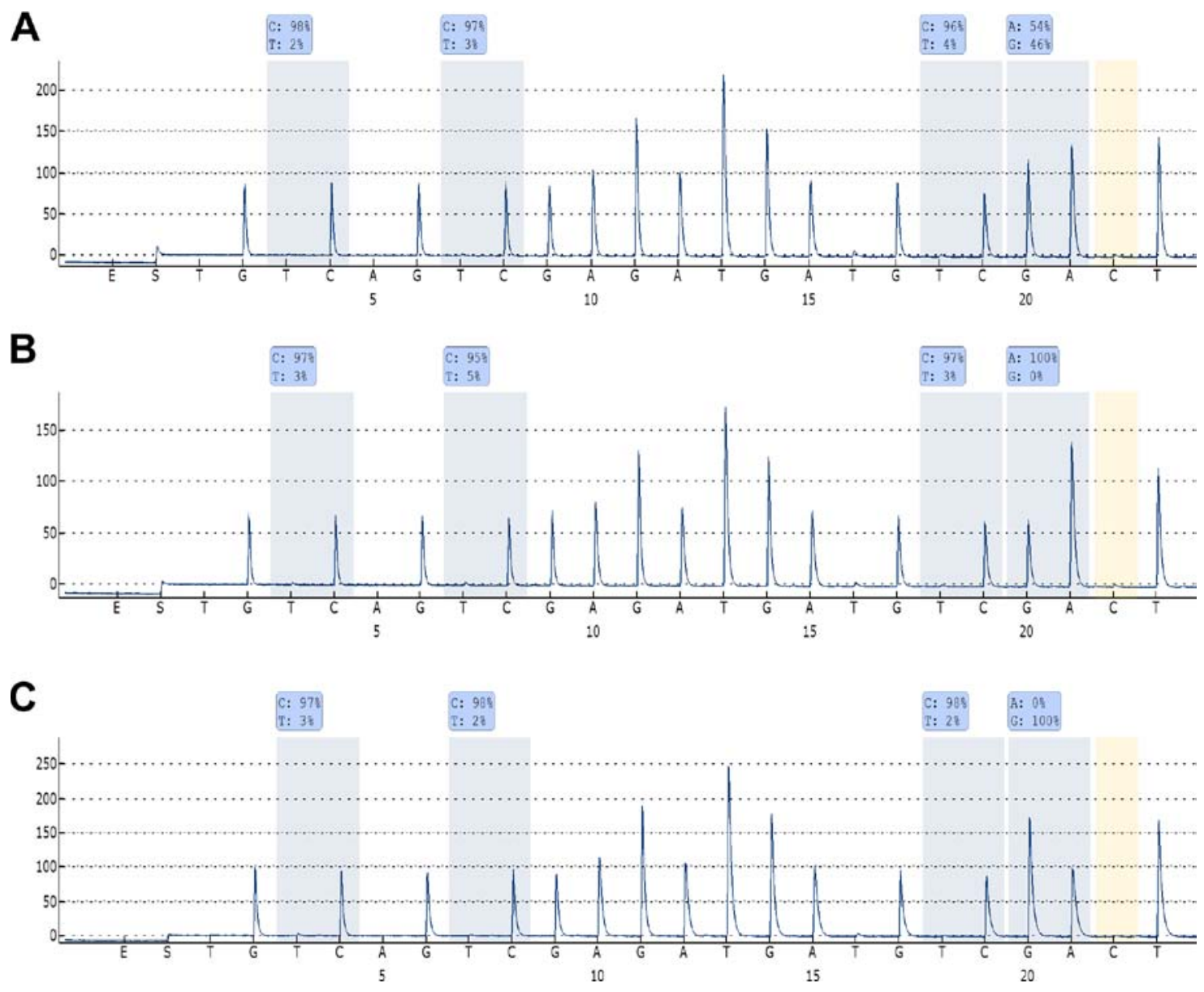

Figure 3: Allelic methylation analyses of DAPK1 in DLBCL. (A) Pyrogram of a sample being methylated at both alleles. (B) Pyrogram of a sample being methylated only at the A-allele. (C) Pyrogram of a sample being methylated only at the G-allele. High methylation levels of the $\mathrm{CpG}$ sites indicate that amplification occurred from methylated template, and it can be observed that the bisulfite conversion of the non- $\mathrm{CpG} \mathrm{C}$ is essentially complete. Thus, these methylation results are regarded as true positive results. 

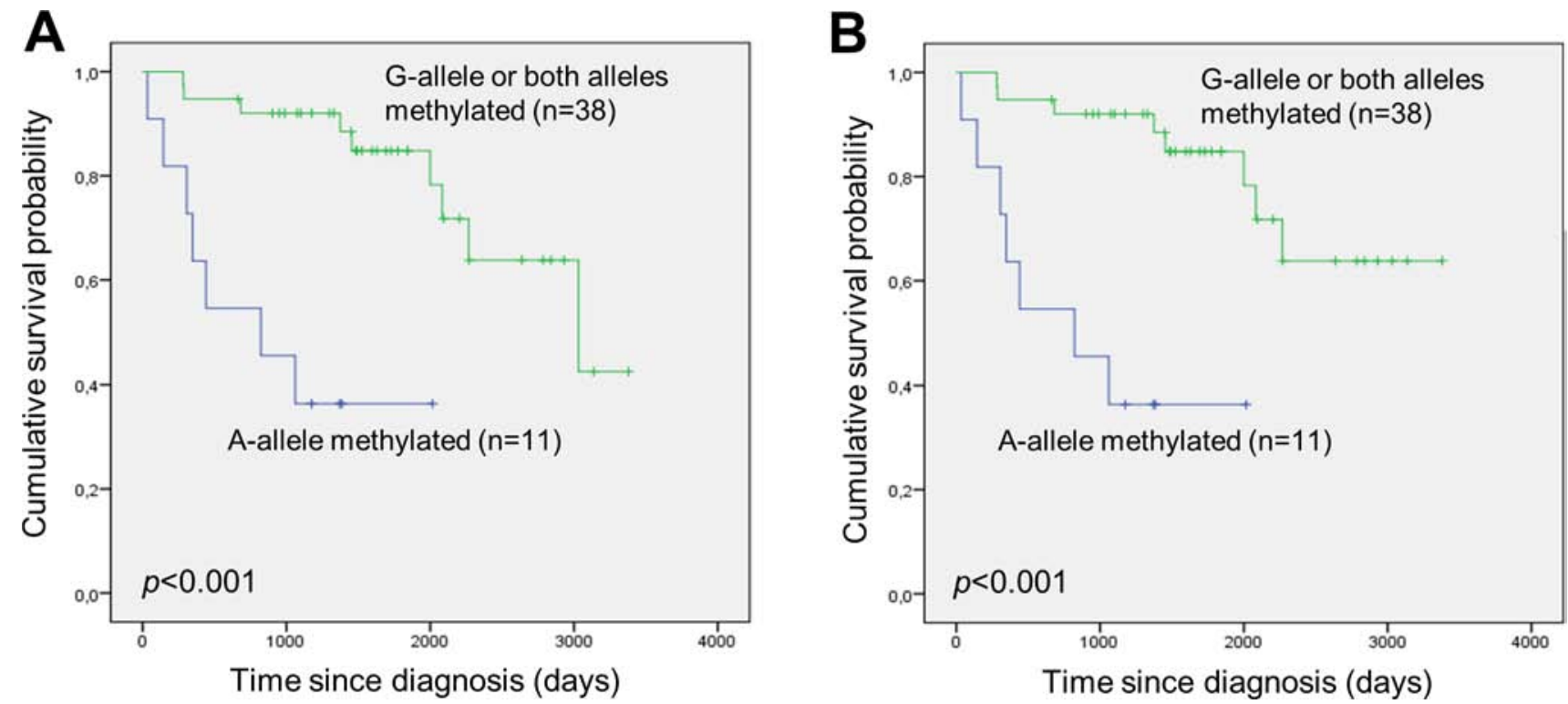

Figure 4: Monoallelic methylation of the A-allele of the rs13300553 DAPK1 promoter SNP is associated with inferior survival in DLBCL. (A) Overall survival according to monoallelic methylation of the A-allele and the other possible allelic methylation patterns. (B) Disease-specific survival according to monoallelic methylation of the A-allele and the other possible allelic methylation patterns.

Table 2: Impact of clinicopathological parameters on disease-specific survival in DLBCL.

\begin{tabular}{|c|c|c|c|}
\hline & Total $(n=119)$ & Number of events & $p$-value \\
\hline \multicolumn{4}{|l|}{ Sex } \\
\hline Men & 58 & 20 & \multirow{2}{*}{0.079} \\
\hline Women & 61 & 12 & \\
\hline \multicolumn{4}{|c|}{ Extranodal involvement } \\
\hline Yes & 57 & 18 & \multirow{2}{*}{0.27} \\
\hline No & 62 & 14 & \\
\hline \multicolumn{4}{|l|}{ Stage } \\
\hline I-II & 51 & 8 & \multirow{2}{*}{0.008} \\
\hline III-IV & 68 & 24 & \\
\hline \multicolumn{4}{|c|}{ Elevated LDH } \\
\hline Yes & 58 & 24 & \multirow{2}{*}{$<0.001$} \\
\hline No & 61 & 8 & \\
\hline \multicolumn{4}{|c|}{ B symptoms } \\
\hline Yes & 48 & 18 & \multirow{2}{*}{0.034} \\
\hline No & 71 & 14 & \\
\hline \multicolumn{4}{|l|}{ IPI score } \\
\hline $0-2$ & 80 & 14 & \multirow{2}{*}{$<0.001$} \\
\hline $3-5$ & 39 & 18 & \\
\hline
\end{tabular}

(Continued) 


\begin{tabular}{|l|c|c|c|}
\hline \multicolumn{2}{c|}{ Total (n=119) } & & \multirow{2}{*}{$p$-value } \\
\hline Performance score* & & & \multirow{2}{*}{0.023} \\
\hline $0-2$ & 113 & 4 & \\
\hline $3-5$ & 6 & & \multirow{2}{*}{0.048} \\
\hline Age at diagnosis & & 13 & \\
\hline Below 65 years & 67 & 19 & \\
\hline Above 65 years & 52 & & \\
\hline
\end{tabular}

"Eastern Cooperative Oncology Group

LDH, lactate dehydrogenase; IPI, international prognostic index.

Table 3: Multivariate Cox regression analyses for prognostic factors affecting disease-specific survival of the DLBCL patients.

\begin{tabular}{|c|c|c|c|c|}
\hline & \multirow[t]{2}{*}{ Hazard ratio } & \multicolumn{2}{|c|}{ 95\% Hazard Ratio Confidence Limits } & \multirow[t]{2}{*}{$p$-value } \\
\hline & & Lower & Upper & \\
\hline \multicolumn{5}{|l|}{ All patients $(n=119)$} \\
\hline Stage (III-IV) & 1.971 & 0.747 & 5.196 & 0.170 \\
\hline Elevated LDH & 4.584 & 1.776 & 11.834 & 0.002 \\
\hline B symptoms & 0.850 & 0.374 & 1.930 & 0.698 \\
\hline IPI score (3-5) & 1.156 & 0.419 & 3.186 & 0.779 \\
\hline Performance score (poor) & 1.117 & 0.347 & 3.601 & 0.853 \\
\hline$D A P K 1$ methylation (yes) & 8.429 & 1.130 & 62.878 & 0.038 \\
\hline \multicolumn{5}{|l|}{$\begin{array}{l}\text { Methylated and } \\
\text { heterozygous patients }(n=49)\end{array}$} \\
\hline Elevated LDH & 7.761 & 1.514 & 39.778 & 0.014 \\
\hline IPI score $(3-5)$ & 2.955 & 0.907 & 15.185 & 0.068 \\
\hline Allelic methylation (A-allele) & 3.103 & 0.901 & 10.691 & 0.073 \\
\hline
\end{tabular}

evaluated for allelic methylation patterns (heterozygous for the rs13300553 SNP and/or unmethylated). When assessing the expression levels among these samples no clear picture emerged. DAPK1 was expressed at very low levels in two unmethylated samples, and at similar levels in samples methylated on both alleles as in samples methylated on one allele, with the exception of one sample (Figure 5B). No RT-enzyme control and no template control were negative.

\section{Overall- and disease-specific survival analysis according to DAPK1 methylation and TP53 mutation status}

TP53 mutation status was obtained for 117 of the DLBCL patients (Supplementary Table 1), and 30 were shown to carry a TP53 mutation (25.6\%). Patients with a TP53 mutation had an inferior overall- and disease-specific survival compared to wild-type patients, however, this was not statistically significant (Supplementary Figure 3A and 3B). Patients carrying both $D A P K 1$ methylation and a TP53 mutation had an inferior overall- and diseasespecific survival compared to patients carrying only one of these molecular alterations, however, this was borderline statistically significant (Figure 6). Finally, no association between TP53 mutational status and DAPK1 methylation was observed $(p=0.77)$.

\section{Allelic DAPK1 methylation patterns in multiple myeloma}

Fifteen of the 67 multiple myeloma patient samples analysed were methylated (22\%). Among the 15 methylated samples six were heterozygous for the rs13300553 SNP. Pyrosequencing was successful for five of these samples, which were all methylated on both alleles (Supplementary Figure 4A). For the remaining sample amplification occurred mainly from the G-allele (80\%), however, the pyrosequencing results were uncertain due 

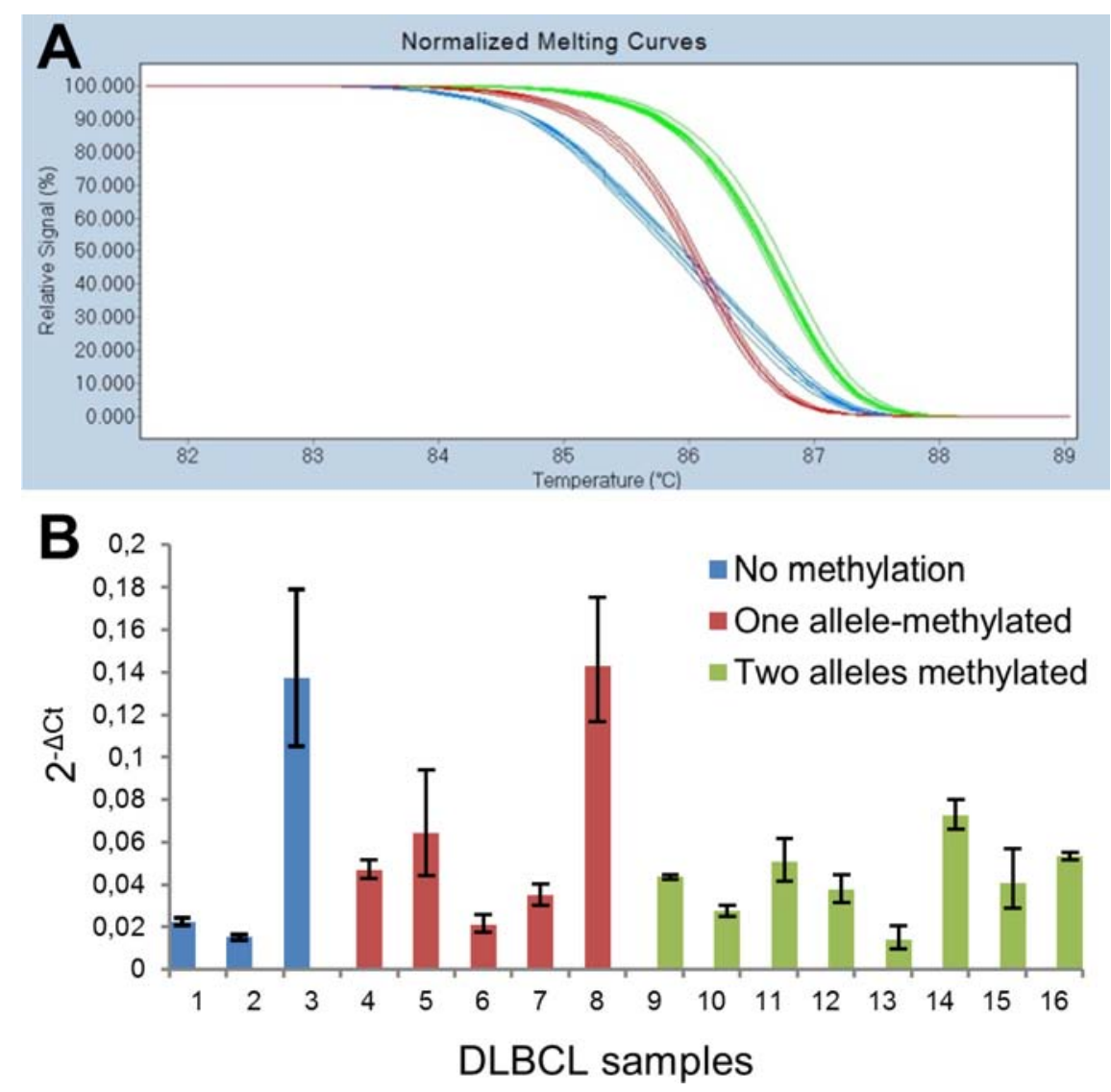

Figure 5: Allele-specific and quantitative expression analyses of $\boldsymbol{D A P K 1}$. (A) HRM analysis of the rs3818584 SNP revealed all 14 heterozygous patients expressed both alleles (blue curves). For the homozygous patients (green and red curves) the HRM analysis was identical to genotyping results. (B) Quantitative assessment of DAPK1 expression in samples for which allelic methylation patterns were analysed. The error bars indicate the highest and lowest possible expression estimates, which can be obtained from the raw Ct-values for $D A P K 1$ and the reference gene, PUM1.

to low peak heights (Supplementary Figure 4B). Also, the methylation specific PCR resulted in a weak band when performing gel electrophoresis for this sample, indicating that the methylation level was low. Therefore, precise quantification of the methylated alleles was difficult for this sample. No association between overall- and diseasespecific survival according to $D A P K 1$ methylation was observed within this patient cohort (data not shown).

\section{DISCUSSION}

It has been postulated that DAPK1 methylation is likely to be a prognostic factor that could be used in conjunction with the conventional prognostic factors such as the IPI score [10]. However, another study did not find a correlation between methylation status of $D A P K 1$ and the prognosis of DLBCL patients [11]. Common for these two studies is that the patient cohorts were relatively small (46 and 53 patients, respectively), and not all patients received treatment with rituximab. We have also previously analysed $D A P K 1$ methylation patterns in a cohort of 74 uniformly treated DLBCL patients [13].
In this study $D A P K 1$ methylation was not statistically significantly associated with overall survival despite none of the patients without DAPK1 methylation died during the follow-up period. Because of these limitations, and because R-CHOP is now standard treatment for patients with DLBCL we increased the cohort to a total of 119 uniformly treated patients with additional follow-up time to investigate whether $D A P K 1$ methylation can predict overall- and disease-specific survival in DLBCL. Similar to previous studies we used MSP for the detection of $D A P K 1$ methylation. However, since MSP is prone to false-positive results [20-23], we used pyrosequencing to confirm all positive results.

Our results confirm the potential of $D A P K 1$ methylation as a prognostic marker in DLBCL treated with $\mathrm{R}-\mathrm{CHOP}$, as it was associated with shorter overall survival $(p=0.017)$ and disease-specific survival $(p=0.023)$. In multivariate analysis $D A P K 1$ methylation remained an independent prognostic factor for predicting diseasespecific survival ( $p=0.038$ ). Furthermore, we observed that, among methylated individuals, monoallelic methylation of the A-allele of the rs13300553 SNP was associated with inferior overall survival $(p<0.001)$ and disease-specific 

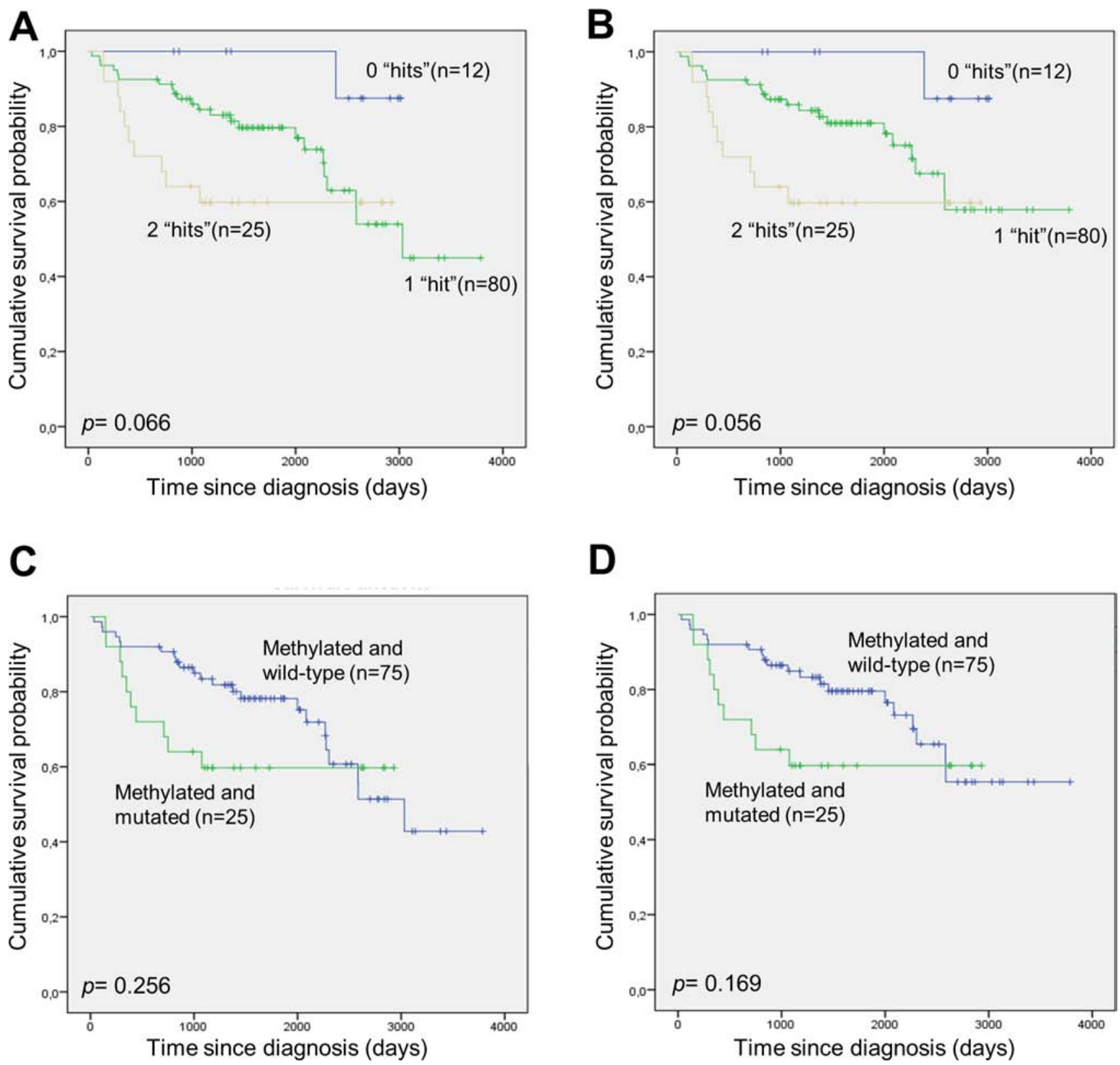

Figure 6: Survival of DLBCL patients with and without DAPK1 methylation and TP53 mutation. (A and B) The patients were divided into three groups, one group without DAPK1 methylation and without TP53 mutation (" 0 hits"), one group with either DAPK1 methylation or TP53 mutation ("1 hit"), and one group with DAPK1 methylation and TP53 mutation ("2 hits"). (A) Overall survival. (B) Disease-specific survival. (C and D) The patients were divided into two groups, one group with DAPK1 methylation and without TP53 mutation, and one group with DAPK1 methylation and TP53 mutation. (C) Overall survival. (D) Disease-specific survival.

survival $(p<0.001)$ as compared to the other possible allelic methylation patterns. This is consistent with the finding that monoallelic methylation of the A-allele was associated with elevated LDH $(p=0.007)$, suggesting that DLBCL with monoallelic methylation of the A-allele are more aggressive. These observations were surprising as we hypothesized that biallelic methylation would be a more severe event compared to monoallelic methylation.

A possible explanation for this phenomenon could be that the rs13300553 SNP or another genetic alteration acting in cis, predispose the allele to become methylated resulting in a constitutional epimutation [24]. This has for instance been observed for the $\mathrm{MSH} 2$ gene in families with Lynch syndrome [25], and, interestingly, in chronic lymphocytic leukemia (CLL) allele-specific expression of DAPK1 has been shown to occur in non-malignant cells in more than $10 \%$ of CLL cases but not in a healthy control population. Moreover, this finding was associated with earlier onset $(p=0.044)$ suggesting a potential mechanism for predisposition to CLL $[26,27]$. Wei and colleagues also showed that allele-specific expression is correlated with elevated 
methylation levels in CLL [27], however, it is unlikely that a genetic alteration acting in cis predispose the allele to become methylated as no genetic aberrations could be identified in spite of extensive upstream and downstream sequencing [27]. We analysed allele-specific expression of $D A P K 1$ in 14 DLBCL samples from patients heterozygous for the rs3818584 SNP using HRM analysis of RT-qPCR products containing this SNP. In spite several of these patients carried monoallelic methylation, we observed a robust expression from both alleles in all samples. However, the signal is likely to be derived from normal cells within the tissue sections. Therefore, we believe that germline allele-specific expression is unlikely to play a significant role in DLBCL pathogenesis. Furthermore, we did not observe any associations between allelic methylation patterns and age at the time of diagnosis.

When analysing the genotypes of the rs 13300553 SNP it could be observed that, within this patient cohort, it did not influence survival by itself, and it does not affect the amino acid sequence of DAPK1. Therefore, we do not believe that the A-allele by itself influence survival of DLBCL patients.

Another possible explanation could be that the combination of having an $\mathrm{A}$ at the position of the rs13300553 SNP in combination with a methylated allele affects the binding of specific proteins and/or nucleosome positioning at the DAPK1 promoter region. We were, however, not able to study these possibilities as none of the DLBCL cell lines we have screened for allelic DAPKI methylation patterns carried monoallelic methylation [13], but we observed that DAPK1 was expressed at similar levels in samples from patients with monoallelic methylation and patients with biallelic methylation. However, as the tissue sections also contain normal cells, and precise estimation of tumour cell content is difficult [28], it is likely that potential differences remain undetected in a background of normal cells expressing DAPK1. In addition, it is likely that the tumour cells are heterogeneous with respect to DAPK1 methylation and expression, which would also confound the expression analysis. Finally, $D A P K 1$ may also be downregulated by other mechanisms such as microRNA mediated mRNA destruction.

In the multivariate analysis monoallelic methylation of the A-allele did not remain as an independent prognostic factor for predicting disease-specific survival $(p=0.07)$. Also, it was observed that more patients carrying monoallelic methylation of the A-allele were male and had a bad performance score. However, the observed survival differences cannot be solely explained by these factors.

To further investigate a potential role of carrying monoallelic methylation of the A-allele in hematologic cancer, we studied 67 samples from patients with multiple myeloma as DAPK1 has previously been shown to undergo methylation mediated silencing in this disease [29]. Interestingly, all of the methylated and heterozygous myeloma samples carried biallelic methylation and
$D A P K 1$ methylation was not associated with inferior survival in this patient cohort. Thus, DAPK1 methylation may play different roles in the tumorigenesis of different haematological malignancies.

DAPK1 methylation and TP53 mutation status have not previously been investigated simultaneously in a single cohort of DLBCL patients and associated with survival. In our study, patients carrying both DAPK1 methylation and a TP53 mutation had an inferior overalland disease-specific survival compared to patients carrying only DAPK1 methylation, however, this was borderline statistically significant. Furthermore, we did not observe a significant correlation between DAPK1 methylation and TP53 mutations, and TP53 mutation status was not significantly associated with overall- or disease-specific survival within this patient cohort. These observations comply with the findings that DAPK1 may facilitate apoptosis both dependent and independent of $\mathrm{p} 53$, and two previous studies of DAPK1 methylation and TP53 mutations in non-small cell lung cancer also found no associations between these molecular events [30, 31].

In conclusion, we found that DAPK1 methylation is associated with poor overall- and disease-specific survival in a large cohort of DLBCL patients uniformly treated with rituximab. This finding remained statistically significant in multivariate analyses. Moreover, methylation of the A-allele of the rs $13300553 \mathrm{SNP}$ was associated with inferior overall- and disease-specific survival in DLBCL. Germline allele-specific expression of $D A P K 1$ is unlikely to be responsible for the inferior survival associated with methylation of the A-allele. Finally, DLBCL with combined TP53 mutation and DAPK1 methylation is likely to be another "double hit" lymphoma with very poor outcome.

\section{MATERIALS AND METHODS}

\section{Patient samples, DNA isolation, and bisulfite conversion}

This study builds upon two previous studies [13, 15] from which allelic DAPK1 methylation information and TP53 mutation status were available for 74 and 62 of the patients, respectively. Additional cases were subsequently collected and analyzed for these two molecular aberrations. The clinical follow-up period for each patient was also prolonged in this study. In total the cohort comprised of 119 DLBCL cases all receiving immunotherapy with rituximab. The diagnoses were based on standard histology and immunophenotyping according to the 2008 WHO classification. The fraction of tumor cells was more than $50 \%$ of the total tissue for all samples, and more than $80 \%$ for the samples used for expression studies. Cause of death was available for all patients except three. These three patients most likely died from their disease and were analyzed as such. In 
addition, 67 patients diagnosed with multiple myeloma were included in the present study. From these patients freshly isolated mononuclear cells from the bone marrow were used for isolation of CD138+ plasma cells on a RoboSep (Stem Cell Technologies, Vancouver, Canada). DNA isolation and subsequent bisulfite conversion were performed as described [13].

The project has been approved by the regional ethics committee (De Videnskabsetiske Komitéer, Region Hovedstaden), as a register project according to the Danish ethical regulations, and informed consent was provided for each multiple myeloma patient sample.

\section{Allelic MSP-pyrosequencing}

The DAPK1 MSP primers were designed to target the sense strand and amplify the region surrounding the rs13300553 SNP (A/G). Several non-CpG cytosines in each of the primers select against the amplification of incompletely converted molecules. Additional $\mathrm{CpG}$ sites and non-CpG cytosines in between the primers serve as a control for the amplification of methylated and bisulfite converted template, respectively. An assay specific for unmethylated $D A P K 1$ sequences was designed to target the same region to verify that negative samples were negative because they were unmethylated and not because the DNA was lost during bisulfite conversion. Genotyping the rs13300553 SNP was done using M-13 tagged DAPK1 specific PCR primers and sequenced using Sanger sequencing. The primer sequences have been published previously [13]. PCR cycling was performed on the Gene PCR System 9700 (Applied Biosystems). The cycling protocols and concentrations of the reagents used have been published [13]. The PCR amplified DNA was run on 2\% agarose gels and sequenced on the PyroMark Q24 (Qiagen) according to the manufacturers' protocol. The same criteria for scoring the samples were applied as in our previous publication [13].

\section{Detection of TP53 mutations}

The coding sequences and splice sites of exons 5-9 of the TP53 gene were scanned for mutations by PCR and denaturing gradient gel electrophoresis (DGGE). By covering exon 5-9 it is expected that about $90 \%$ of TP53 mutations will be detected [14]. All mutations were confirmed in a second round of PCR from the original sample, and positive samples were subjected to Sanger sequencing as described [15].

\section{Allele-specific and quantitative expression analyses of $D A P K 1$}

RNA was extracted from 24 specimens having at least $80 \%$ tumour cells using the miRNeasy mini kit (Qiagen, Hilden, Germany), which extract both
microRNAs as well as total RNA, according to the manufacturers' protocol including DNAase treatment. From each sample $250 \mathrm{ng}$ RNA was used for cDNA synthesis, which was performed using the SuperScript ${ }^{\circledR}$ III First-Strand Synthesis System (Life Technologies) according to the manufacturers' protocol. The cDNA was diluted 1:10 before real-time PCR. The LightCycler ${ }^{\circledR}$ 480 instrument II (Roche, Mannheim, Germany) was used for the real-time PCR and high-resolution melting (HRM). The High Resolution Melting Master (Roche) was used at a final concentration of $1 \mathrm{X}$, with $2.5 \mathrm{mM}$ $\mathrm{MgCl}_{2}, 200 \mathrm{nM}$ of each primer, and $5 \mu \mathrm{L}$ of diluted cDNA resulting in a total volume of $20 \mu \mathrm{L}$. The cycling protocol started with one cycle of $95^{\circ} \mathrm{C}$ for $10 \mathrm{~min}$, followed by 45 cycles of $95^{\circ} \mathrm{C}$ for $5 \mathrm{~s}, 60^{\circ} \mathrm{C}$ for $10 \mathrm{~s}$, and $72^{\circ} \mathrm{C}$ for $10 \mathrm{~s}, 1$ cycle of $95^{\circ} \mathrm{C}$ for $1 \mathrm{~min}, 1$ cycle of $40^{\circ} \mathrm{C}$ for $1 \mathrm{~min}$, and a melting step from $65^{\circ} \mathrm{C}$ to $95^{\circ} \mathrm{C}$ with 30 acquisitions per ${ }^{\circ} \mathrm{C}$. All reactions were done in duplicate. The sequences of the DAPK1 primers were; forward: 5' Biotin-CAGGGGCTACCACGACAT 3' and reverse: 5' AGGGCAATGTGTCCGTCCTT 3'. The reverse primer was designed to overlap an exonexon junction to prevent amplification of potentially contaminating genomic DNA. The forward primer was biotin labelled to allow confirmation of the HRM data by pyrosequencing, using the PyroMark Q24 (Qiagen) according to the manufacturers' protocol, with the following sequencing primer: 5' GCTTGCGACAAGGAC 3'. The samples were genotyped for the rs3818584 SNP using the same forward primer as above and reverse: 5' CCATAAGGCACCTTGTCGCAA 3' using the PyroMark Q24 (Qiagen) according to the manufacturers' protocol.

For the quantitative analyses the PUM1 gene was used for normalization as this gene has previously been shown to be stably expressed in lymphoid tissue [32]. The primer sequences were; forward: 5' CATGCCAGGTTATCCGGTGT 3' and reverse: 5' GCGCCTGCATTCACTACAAG 3'. The same concentrations of reagents and LightCycler protocol were used as described above. The data were expressed as relative quantities $\left(2^{-\Delta C t}\right)$ using the average of the Ct-value from each of the duplicates. The highest possible estimate for $D A P K 1$ expression for each sample was obtained by using the lowest $D A P K 1 \mathrm{Ct}$-value and the highest PUM1 $\mathrm{Ct}$-value to generate the $\Delta \mathrm{Ct}$-value, and vice versa for the lowest possible estimates.

\section{Statistical analysis}

Statistical analyses were performed in SPSS 19.0 for Windows (SPSS Inc.). Associations between $D A P K 1$ methylation and TP53 mutations were assessed using a Fisher's exact test. Correlations between overall survival and disease specific survival and TP53 mutation status and DAPK1 methylation status were estimated using the Kaplan-Meier method with 
the use of a log-rank test. The clinical characteristics and treatment outcomes were compared according to TP53 mutation status and DAPK1 methylation status using one-way ANOVA, Person chi-squared tests, or Fisher's exact tests when expected values were below five. For assessment of independent predictors of disease specific survival a multivariate Cox regression hazard model with backward stepwise (likelihood ratio) entry was applied. Effects not meeting a $p$-value $<0.05$ in univariate analyses were removed from the model. Any differences were considered to be statistically significant when the $p$-value was $<0.05$.

\section{ACKNOWLEDGEMENTS}

This study was supported by grants from The Novo Nordisk Foundation, Rigshospitalets Research Foundation, The Danish Cancer Society, and The Danish Strategic Research Foundation. The funders had no role in study design, data collection and analysis, decision to publish, or preparation of the manuscript.

\section{Authors' contributions}

FA, MKN, DA, and KD carried out the molecular genetic studies and analysed the data. JWH and FA collected the clinical data. ER classified the DLBCL samples. LSK conceived the study, carried out the molecular genetic studies, analysed the data, and wrote the manuscript. KG participated in the design of the study, contributed reagents and materials, and helped to draft the manuscript. All authors read and approved the final manuscript.

\section{Conflicts of interests} interests.

The authors declare that they have no competing

\section{Editorial note}

This paper has been accepted based in part on peerreview conducted by another journal and the authors' response and revisions as well as expedited peer-review in Oncotarget.

\section{REFERENCES}

1. Herrmann K, Buck AK, Schuster T, Abbrederis K, Blumel C, Santi I, Rudelius M, Wester HJ, Peschel C, Schwaiger M, Dechow T, Keller U. Week one FLT-PET response predicts complete remission to R-CHOP and survival in DLBCL. Oncotarget. 2014; 5:4050-4059.

2. Ezell SA, Mayo M, Bihani T, Tepsuporn S, Wang S, Passino M, Grosskurth SE, Collins M, Parmentier J, Reimer C, Byth KF.
Synergistic induction of apoptosis by combination of BTK and dual mTORC1/2 inhibitors in diffuse large B cell lymphoma. Oncotarget. 2014; 5:4990-5001.

3. Bialik S, Kimchi A. The DAP-kinase interactome. Apoptosis. 2014; 19:316-328.

4. Raveh T, Droguett G, Horwitz MS, DePinho RA, Kimchi A. DAP kinase activates a p19ARF/p53-mediated apoptotic checkpoint to suppress oncogenic transformation. Nature cell biology. 2001; 3:1-7.

5. Craig AL, Chrystal JA, Fraser JA, Sphyris N, Lin Y, Harrison BJ, Scott MT, Dornreiter I, Hupp TR. The MDM2 ubiquitination signal in the DNA-binding domain of p53 forms a docking site for calcium calmodulin kinase superfamily members. Molecular and cellular biology. 2007; 27:3542-3555.

6. Martoriati A, Doumont G, Alcalay M, Bellefroid E, Pelicci PG, Marine JC. dapk1, encoding an activator of a p19ARFp53-mediated apoptotic checkpoint, is a transcription target of p53. Oncogene. 2005; 24:1461-1466.

7. Bialik S, Kimchi A. The death-associated protein kinases: structure, function, and beyond. Annual review of biochemistry. 2006; 75:189-210.

8. Benderska N, Schneider-Stock R. Transcription control of DAPK. Apoptosis. 2014; 19:298-305.

9. Katzenellenbogen RA, Baylin SB, Herman JG. Hypermethylation of the DAP-kinase $\mathrm{CpG}$ island is a common alteration in B-cell malignancies. Blood. 1999; 93: 4347-4353.

10. Amara K, Trimeche M, Ziadi S, Laatiri A, Hachana M, Korbi S. Prognostic significance of aberrant promoter hypermethylation of $\mathrm{CpG}$ islands in patients with diffuse large B-cell lymphomas. Annals of oncology. 2008; 19:1774-1786.

11. Nakamichi I, Tomita Y, Zhang B, Sugiyama H, Kanakura Y, Fukuhara S, Hino M, Kanamaru A, Ogawa H, Aozasa K. Correlation between promoter hypermethylation of GSTP1 and response to chemotherapy in diffuse large B cell lymphoma. Annals of hematology. 2007; 86:557-564.

12. Krajnovic M, Jovanovic MP, Mihaljevic B, Andelic B, Tarabar O, Knezevic-Usaj S, Krtolica K. Hypermethylation of p15 Gene in Diffuse - Large B-Cell Lymphoma: Association with Less Aggressiveness of the Disease. Clinical and translational science. 2014;

13. Kristensen LS, Treppendahl MB, Asmar F, Girkov MS, Nielsen HM, Kjeldsen TE, Ralfkiaer E, Hansen LL Gronbaek K. Investigation of MGMT and DAPK1 methylation patterns in diffuse large B-cell lymphoma using allelic MSP-pyrosequencing. Scientific reports. 2013; 3:2789.

14. Xu-Monette ZY, Moller MB, Tzankov A, Montes-Moreno S, $\mathrm{Hu}$ W, Manyam GC, Kristensen L, Fan L, Visco C, Dybkaer K, Chiu A, Tam W, Zu Y, Bhagat G, Richards KL, Hsi ED. MDM2 phenotypic and genotypic profiling, respective to TP53 genetic status, in diffuse large B-cell 
lymphoma patients treated with rituximab-CHOP immunochemotherapy: a report from the International DLBCL Rituximab-CHOP Consortium Program. Blood. 2013; 122:2630-2640.

15. Asmar F, Hother C, Kulosman G, Treppendahl MB, Nielsen HM, Ralfkiaer U, Pedersen A, Moller MB, Ralfkiaer E, de Nully Brown P, Gronbaek K. Diffuse large B-cell lymphoma with combined TP53 mutation and MIR34A methylation: Another "double hit" lymphoma with very poor outcome? Oncotarget. 2014; 5:1912-1925.

16. Jardin F, Jais JP, Molina TJ, Parmentier F, Picquenot JM, Ruminy P, Tilly H, Bastard C, Salles GA, Feugier P, Thieblemont C, Gisselbrecht C, de Reynies A, Coiffier B, Haioun C, Leroy K. Diffuse large B-cell lymphomas with CDKN2A deletion have a distinct gene expression signature and a poor prognosis under R-CHOP treatment: a GELA study. Blood. 2010; 116:1092-1104.

17. Gronbaek K, de Nully Brown P, Moller MB, Nedergaard T, Ralfkiaer E, Moller P, Zeuthen J, Guldberg P. Concurrent disruption of p16INK4a and the ARF-p53 pathway predicts poor prognosis in aggressive non-Hodgkin's lymphoma. Leukemia. 2000; 14:1727-1735.

18. Kristensen LS, Treppendahl MB, Gronbaek K. Analysis of epigenetic modifications of DNA in human cells. Current protocols in human genetics. 2013; Chapter 20:Unit20 22.

19. Kristensen LS, Hansen LL. PCR-based methods for detecting single-locus DNA methylation biomarkers in cancer diagnostics, prognostics, and response to treatment. Clinical chemistry. 2009; 55:1471-1483.

20. Shaw RJ, Akufo-Tetteh EK, Risk JM, Field JK, Liloglou T. Methylation enrichment pyrosequencing: combining the specificity of MSP with validation by pyrosequencing. Nucleic acids research. 2006; 34:e78.

21. Kristensen LS, Mikeska T, Krypuy M, Dobrovic A. Sensitive Melting Analysis after Real Time- Methylation Specific PCR (SMART-MSP): high-throughput and probe-free quantitative DNA methylation detection. Nucleic acids research. 2008; 36:e42.

22. Kristensen LS, Raynor MP, Candiloro I, Dobrovic A. Methylation profiling of normal individuals reveals mosaic promoter methylation of cancer-associated genes. Oncotarget. 2012; 3:450-461.

23. Rand K, Qu W, Ho T, Clark SJ, Molloy P. Conversionspecific detection of DNA methylation using real-time polymerase chain reaction (ConLight-MSP) to avoid false positives. Methods. 2002; 27:114-120.
24. Dobrovic A, Kristensen LS. DNA methylation, epimutations and cancer predisposition. The international journal of biochemistry \& cell biology. 2009; 41:34-39.

25. Ligtenberg MJ, Kuiper RP, Chan TL, Goossens M, Hebeda KM, Voorendt M, Lee TY, Bodmer D, Hoenselaar E, Hendriks-Cornelissen SJ, Tsui WY, Kong CK, Brunner HG, van Kessel AG, Yuen ST, van Krieken JH. Heritable somatic methylation and inactivation of MSH2 in families with Lynch syndrome due to deletion of the 3' exons of TACSTD1. Nature genetics. 2009; 41:112-117.

26. Raval A, Tanner SM, Byrd JC, Angerman EB, Perko JD, Chen SS, Hackanson B, Grever MR, Lucas DM, Matkovic JJ, Lin TS, Kipps TJ, Murray F, Weisenburger D, Sanger W, Lynch J. Downregulation of death-associated protein kinase 1 (DAPK1) in chronic lymphocytic leukemia. Cell. 2007; 129:879-890.

27. Wei QX, Claus R, Hielscher T, Mertens D, Raval A, Oakes CC, Tanner SM, de la Chapelle A, Byrd JC, Stilgenbauer $\mathrm{S}$, Plass C. Germline allele-specific expression of DAPK1 in chronic lymphocytic leukemia. PloS one. 2013; 8:e55261.

28. Lade-Keller J, Romer KM, Guldberg P, Riber-Hansen R, Hansen LL, Steiniche T, Hager H, Kristensen LS. Evaluation of BRAF mutation testing methodologies in formalin-fixed, paraffin-embedded cutaneous melanomas. The Journal of molecular diagnostics. 2013; 15:70-80.

29. Seidl S, Ackermann J, Kaufmann H, Keck A, Nosslinger T, Zielinski CC, Drach J, Zochbauer-Muller S. DNA-methylation analysis identifies the E-cadherin gene as a potential marker of disease progression in patients with monoclonal gammopathies. Cancer. 2004; 100:2598-2606.

30. Liu Y, Gao W, Siegfried JM, Weissfeld JL, Luketich JD, Keohavong P. Promoter methylation of RASSF1A and DAPK and mutations of K-ras, p53, and EGFR in lung tumors from smokers and never-smokers. BMC cancer. 2007; 7:74.

31. Kim DH, Nelson HH, Wiencke JK, Christiani DC, Wain JC, Mark EJ, Kelsey KT. Promoter methylation of DAP-kinase: association with advanced stage in non-small cell lung cancer. Oncogene. 2001; 20:1765-1770.

32. Soes S, Sorensen BS, Alsner J, Overgaard J, Hager H, Hansen LL, Kristensen LS. Identification of accurate reference genes for RT-qPCR analysis of formalin-fixed paraffin-embedded tissue from primary non-small cell lung cancers and brain and lymph node metastases. Lung Cancer. 2013; 81:180-186. 